\title{
A Volume Product Representation and Its Ramifications in $I_{p}^{n}, 1 \leq p \leq \infty$
}

\author{
Dimitris Karayannakis \\ Department of Science, Division of Mathematics, TEI of Crete, Heraklion, Greece \\ E-mail:dkar@staff.teicrete.gr
}

Received April 19, 2011; revised June 9, 2011; accepted June 20, 2011

\begin{abstract}
Let $\left|B_{p}^{n}\right|, 1<p<\infty$, be the volume of the unit $p$-ball in $R^{n}$ and $q$ the Hölder conjugate exponent of $p$. We represent the volume product $\left|B_{p}^{n}\right|\left|B_{q}^{n}\right|$ as a function free of its gamma symbolism. This representation will allows us in this particular case to confirm, using basic classical analysis tools, two conjectured and partially proved lower and upper bounds for the volume product of centrally symmetric convex bodies of the Euclidean $R^{n}$. These bounds in the general case play a central role in convex geometric analysis.
\end{abstract}

Keywords: P-Ball, Volume, Gamma Function, Infinite Product

\section{Introduction}

One of the key notions in convex analysis is the volume product $M(K)=|K|\left|K^{o}\right|$ where $K$ is a (centrally symmetric) convex compact set of the Euclidean $\left(R^{n},\langle.,\rangle.\right)$ with non-empty interior (or simply "body") and $K^{o}=\left\{y \in R^{n}:|\langle x, y\rangle| \leq 1, \forall x \in K\right\}$ is the so called polar set of $K$

For this product, one of the long standing conjectures stated by Mahler (who proved it for $n=2$ ) claims that $\frac{4^{n}}{n !} \leq M(K)$ for origin symmetric bodies $K$. This conjecture has been confirmed in many special cases of $K$ and in particular for bodies symmetric with respect to the coordinate planes, which naturally include the $p$-balls, using a rather advanced Banach space theory. On the other hand, for an upper bound, we have the inequality $M(K) \leq M\left(B_{2}^{n}\right)$ proved in 1948 by Santaló (and much earlier for $n=2$ by Blashke). A survey of the above facts and other related results can be found e.g. in [4].

We set forward in this short work to establish Mahler's conjecture and the Blashke-Santaló inequality for the case $K=B_{p}^{n}, 1 \leq p \leq \infty$, using exclusively basic special functions and classical analysis theory.

It is clear that $\left(B_{p}^{n}\right)^{o}=B_{q}^{n}$, where $\frac{1}{p}+\frac{1}{q}=1$, and also directly verifiable through multiple integration (see e.g. [1]) that $\left|B_{p}^{n}\right|=\frac{2^{n} \Gamma\left(1+\frac{1}{p}\right)^{n}}{\Gamma\left(1+\frac{n}{p}\right)}$ for $1<p<\infty$ and $\left|B_{1}^{n}\right|=\left|B_{\infty}^{n}\right|=2^{n}$.

So we now have to manipulate the expression

$$
M\left(B_{p}^{n}\right)=4^{n} \frac{\left[\Gamma\left(1+\frac{1}{p}\right) \Gamma\left(1+\frac{1}{q}\right)\right]^{n}}{\Gamma\left(1+\frac{n}{p}\right) \Gamma\left(1+\frac{n}{q}\right)} .
$$

Thus, in Sec.2 we start with a suitable for our goals gamma functions ratio result (Lemma) that will allow us to represent $M\left(B_{p}^{n}\right)$ as a suitable function $M(n, p)$, for $1<p<\infty$, free of its gamma symbolism (Proposition); finally, in Sec.3, by establishing $\frac{\mathrm{d} M(n, p)}{\mathrm{d} p} \geq 0$ for $1<p \leq 2$ (Lemma) and then using the evident facts that $M(n, p)=M(n, q)$ and $1<q \leq 2$ iff $2 \leq p<\infty$, and also by examining separately the case $p=1$, we will obtain the announced results (Proposition). We also obtain, as byproducts of independent interest, three seemingly new closed formulae concerning infinite products (Corol. 1, Corol. 2 and Rem. 2). 


\section{A Lemma and a Proposition}

\section{Lemma}

For $x>0$ and $0<\alpha<1$ we have that

$$
\Gamma(1-\alpha) \frac{\Gamma(x+\alpha)}{\Gamma(x)}=\prod_{k=1}^{\infty} \frac{k(k+x-1)}{(k-\alpha)(k+x+\alpha-1)} .
$$

Proof:

Let $P(x, \alpha)$ be the infinite product in lemma's statement .One of the classical definitions for the gamma function for any complex $z \neq 0,-1,-2, \cdots$, is

$$
\Gamma(z)=\lim _{k \rightarrow \infty} \frac{k^{z-1} k !}{(z)_{k}},
$$

where $(z)_{k}$ is the shifted factorial $z(z+1) \cdots(z+k-1)$.Substituting in the above limit, respectively, $z=1-\alpha, z=x+\alpha$, and $z=x$, and after simplifying we arrive at

$\Gamma(1-\alpha) \frac{\Gamma(x+\alpha)}{\Gamma(x)}=\lim _{k \rightarrow \infty} \frac{k !(x)_{k}}{(1-\alpha)_{k}(x+\alpha)_{k}}$ which is identical to $P(x, \alpha)$.

\section{Remark}

1) Independently of the above argumentation we can easily check that $P(x, \alpha)$ exists as a two-variable funcction over $(0, \infty) \times[0,1)$ since evidently $0 \leq P(x, \alpha)$ and, by use of the inequality $\log t \leq t-1, t>0$,

$$
\begin{aligned}
& \log P(x, \alpha) \\
& \leq \alpha(x+\alpha-1) \sum_{k=1}^{\infty} \frac{1}{(k-\alpha)(k+x+\alpha-1)}<\infty .
\end{aligned}
$$

2) This lemma was proved formally (and from "scratch") in [3] where the scheme of the proof served different purposes concerning the numerical evaluation of the gamma function.

\section{Proposition}

$$
\text { For } 1<p<\infty \text {, }
$$

$$
\begin{aligned}
& M(n, p)= \\
& 4^{n} h(p)^{2 n-2} \prod_{k=1}^{\infty} \frac{\left(k^{2}+n k+\frac{n^{2}}{p q}\right)\left(k^{2}+k+\frac{1}{p q}\right)^{n-2}}{(k+1)^{2 n-2}}
\end{aligned}
$$

where $h(p)=\frac{\pi}{p q \sin \left(\frac{\pi}{p}\right)}$.

Proof:

Evidently $h\left(1^{+}\right)=1$ and so (2) is trivially true for $n=1$. Thus we can consider $n \geq 2$.

At first let us rewrite (1) as

$$
M(n, p)=\frac{4^{n}}{n^{2}} \frac{1}{(p q)^{n-1}} \frac{\left[\Gamma\left(\frac{1}{p}\right) \Gamma\left(\frac{1}{q}\right)\right]^{n}}{\Gamma\left(\frac{n}{p}\right) \Gamma\left(\frac{n}{q}\right)}
$$

Based on Lemma II. 1 for $\alpha=\frac{1}{p}$ and $x=\frac{m}{p}, m=1, \cdots, n-1$ we see that

$$
\Gamma\left(\frac{n}{p}\right)=\frac{\Gamma\left(\frac{1}{p}\right)}{\Gamma\left(\frac{1}{q}\right)^{n-1}} \prod_{m=1}^{n-1} P\left(\frac{n-m}{p}, \frac{1}{p}\right)
$$

where $P(x, \alpha)$ was defined above in Lemma

Working in a similar way we obtain the "conjugate expression" $\Gamma(n / q)$.

We observe now that

$$
\begin{aligned}
& P\left(\frac{n-m}{p}, \frac{1}{p}\right) P\left(\frac{n-m}{q}, \frac{1}{q}\right) \\
& =\frac{1}{p q}\left(\frac{n-m}{n+1-m}\right)^{2} \prod_{k=1}^{\infty} \frac{(k+1)^{2}}{g_{k}(p)} \frac{r_{k}(n-m, p)}{r_{k}(n+1-m, p)}
\end{aligned}
$$

where we have set $r_{k}(j, p)=k^{2}+j k+\frac{j^{2}}{p q}$ and

$g_{k}(p)=r_{k}(1, p)$

Combining (2), (4), and (5), and by "telescoping", we obtain (3).

\section{One More Lemma and a Conclusive Proposition}

\section{Lemma}

Let $1<p<2$. Then $\frac{\mathrm{d} M(n, p)}{\mathrm{d} p} \geq 0$ for all $n$ with equality iff $p=2$ for $n \neq 1$.

Proof:

$M(1, p)=4^{n}$ and once more we can consider $n \geq 2$. By straightforward differentiation with respect to $p$ and in the case of the infinite product of (2) by logarithmic differentiation (noticing that this product by construction is a real analytic function of $p$ having as logarithm a uniformly converging series of differentiable functions of $p$ ) we obtain

$$
\begin{aligned}
& \operatorname{sign}\left[\frac{\mathrm{d} M(n, p)}{\mathrm{d} p}\right] \\
& =\operatorname{sign}\left[(2 n-2) h^{\prime}+\left(\frac{1}{p q}\right)^{\prime} h \sum_{k=1}^{\infty}\left(\frac{n-2}{g_{k}}+\frac{n^{2}}{r_{k}}\right)\right] .
\end{aligned}
$$


In (6) $h=h(p)$ was defined in (3) and

$g_{k}=g_{k}(p), r_{k}=r_{k}(n, p)$ as defined in (5); still for simplicity from now on we suppress any possible dependence from $p$ and $n$.

Since evidently $h$ and the infinite sum in (6) are positive and $(p q)^{\prime}=\frac{p(p-2)}{(p-1)^{2}} \leq 0$ (with equality iff $p=2$ ) it will suffice to show that $h^{\prime} \geq 0$ (with equality iff $p=2$ ).

Simple calculations show that

$$
\begin{aligned}
& \operatorname{signh}^{\prime}=-\operatorname{sign}\left[p q \sin \left(\frac{\pi}{p}\right)\right]^{\prime}=\operatorname{sign}(\omega), \text { where } \\
& \omega=\omega(p)=p(2-p) \sin \left(\frac{\pi}{p}\right)+\pi(p-1) \cos \left(\frac{\pi}{p}\right) .
\end{aligned}
$$

Now

$$
\begin{aligned}
\operatorname{sign}\left(\omega^{\prime}\right) & =\operatorname{sign}\left[\pi(1-p) \sin \left(\frac{\pi}{p}\right)\right. \\
& \left.+\left(p^{2}+2 p-2\right) \cos \left(\frac{\pi}{p}\right)\right]<0,
\end{aligned}
$$

and since $\omega$ we conclude that $\omega \geq 0$ with equality iff $p=2$.

We are now ready to prove the announced double inequality concerning $M(n, p)$.

\section{Proposition}

For $1 \leq p \leq \infty$ and all positive integers $n$,

$\frac{4^{n}}{n !} \leq M(n, p) \leq M(n, 2)$ with left (resp. right) equality iff $p=1$ (resp. $p=2)$, whenever $n \neq 1$.

Proof:

Based on Lemma III.1 we can see immediately that for $1<t<p<\infty, M(n, t)<M(n, p) \leq M(n, 2)$, with equality iff $p=2$.

We only need to examine (2) whenever $p \rightarrow 1^{+}$:

It is clear that $M\left(n, 1^{+}\right)=4^{n} s_{n}$, with

$s_{n}=\prod_{k=1}^{\infty} \frac{k^{n-1}}{(k+1)^{n}}(k+n)$. Since $s_{1}=1$ and

$\frac{s_{n+1}}{s_{n}}=\prod_{k=1}^{\infty}\left(\frac{k}{k+1}\right)\left(\frac{k+n+1}{k+n}\right)=\frac{1}{n+1}$ we see that

$s_{n}=\frac{1}{n !}$ and we are done.

\section{Remark 1}

Independently of the above approach one can easily verify that $s_{n}$ converges for all $n$ by the logarithmic series test along the lines of Remark II. 1(i): evidently $s_{n}>0$ and also for $n \geq 2$ (by the crude logarithmic inequality) $\log s_{n}<\sigma_{n}$, where

$$
\sigma_{n}=\sum_{m=2}^{n}\left(\begin{array}{l}
n \\
m
\end{array}\right) \sum_{k=1}^{\infty} \frac{k^{n-m}}{(k+1)^{n}}<\infty .
$$

Thus we obtain the (crude but not obvious) inequality $\frac{1}{n !}<e^{-\sigma_{n}}$ for $n \geq 2$.

We conclude this work with two closed type formulae that are automatically true when we set $p=2$ in (2). Exploiting the fact that $M(n, 2)=\left|B_{2}^{n}\right|=\frac{4 \pi^{n}}{n^{2} \Gamma^{2}\left(\frac{n}{2}\right)}$ and the classical value of $\Gamma\left(\frac{n}{2}\right)$ for even and odd $n$ (see e.g.[2]) we arrive, respectively, for any $m \in N$ at the following fomulae:

\section{Corollary 1}

$$
\prod_{k=1}^{\infty} \frac{(2 k+1)^{2 m-2}(2 k+2 m)}{(2 k+2)^{2 m-1}}=\frac{\left(\frac{4}{\pi}\right)^{m-1}}{m !}
$$

\section{Corollary 2}

$$
\prod_{k=1}^{\infty} \frac{(2 k+1)^{2 m-1}(2 k+2 m+3)}{(2 k+2)^{2 m}}=\frac{\left(\frac{8}{\pi}\right)^{m+1}}{(2 m+3) ! !}
$$

\section{Remark 2}

It is clear that the above two formulae provide us with the seemingly untabulated formula

$$
\prod_{k=1}^{\infty} \frac{(2 k+2)(2 k+2 m)}{(2 k+1)(2 k+2 m+3)}==\frac{(2 m+3) ! !}{2^{m+1} m !} .
$$

\section{References}

[1] G. Andrews, R. Askey and R. Roy, "Special Functions," Cambridge University Press, Cambridge, 1999.

[2] I. Gradshteyn and I. Ryzhik, "Tables of Integrals, Series and Products," Academic Press, Waltham, 1980.

[3] D. Karayannakis, "An algorithm for the Evaluation of the Gamma function and Ramifications. Part I," International Journal of Mathematics, Game Theory and Algebra, Vol. 19, No. 4, 2010

[4] L. E. Lutwak, "Selected Affine Isoperimetric Inequalities, Handbook of Convex Geometry," North-Holland Publishing Co., Amsterdam, 1993. 